Editorial

\title{
Acknowledgement to Reviewers of Agriculture in 2017
}

\author{
Agriculture Editorial Office \\ MDPI AG, St. Alban-Anlage 66, 4052 Basel, Switzerland
}

Published: 22 January 2018

Peer review is an essential part in the publication process, ensuring that Agriculture maintains high quality standards for its published papers. In 2017, a total of 101 papers were published in the journal. Thanks to the cooperation of our reviewers, the median time to first decision was 27 days and the median time to publication was 64 days. The editors would like to express their sincere gratitude to the following reviewers for their time and dedication in 2017:

Abidi, Khalid

Alcaraz-Quiles, Francisco José

Aleksić, Ana

Alsaaty, Falih

Amery, Fran

Andrews, Rhys

Anonson, June

Antunes, Pedro

Arkoubi, Khadija Al

Bang, Hyochoong

Bariviera, Aurelio F.

Baumann, Andrea

Belias, Dimitrios

Bellasio, Roberto

Bellman, Beryl

Belso-Martínez, José Antonio

Berry, Lois

Bockarie, Abu

Bokhari, Hasnain

Bolzani, Daniela

Bowles, Hannah Riley

Boyer, Teresa

Bramley, Glen

Breitsamter, Christian

Brett, Jeanne

Browne, Liz

Bryant, Phillip

Bygrave, Constance E.

Caetano, António
Campbell, David

Cao, Xin

Carrera, Erasmo

Cerchione, Roberto

Chasalevris, Athanasios

Chen, Ziguang

Cheong, Joono

Chiodo, Elio

Clarke, Sean P.

Cobano, J. A.

Cornelius, Nelarine

Corvo, Luigi

Coskun, Bulut

Daerden, Frank

Dagdeviren, Canan

Debéda, Hélène

Deng, Mingcong

Di, Long

Dörfler, Viktor

Dubois, Fabien

Duchscher, Judy Boychuk

Eckert, Paulo Roberto

Eddy, Pamela L.

Ehrenhard, Michel

Eisler, Karen

Eley, Rob

Englmaier, Florian

Entwistle, Tom

Epple, Philipp 
Felton, Samuel M.

Finegan, Joan

Fisher, Virginia

FitzGerald, Gerry

Fletcher, Luke

Flynn, Anthony

Forbes, Sarah

$\mathrm{Fu}, \mathrm{Chu}-\mathrm{Chun}$

$\mathrm{Fu}$, Xin

Fuller, Kay

Gagnon, Louis

Gallo, Maria

Gamble, Brenda

Gaviria López, Alberto

Gejji, Rohan

Ghodke, Chaitanya

Go Jefferies, Josephine

Goebel, Dan M.

Grandy, Gina

Groothuis, Stefan

Gu, Guoying

Gudmundsson, Snorri

Gunnarsdottir, Sigrun

Gupta, Aniruddha

Han, Bangcheng

Hannibal, Claire

Hartner-Tiefenthaler, Martina

Hatzfeld, Christian

Henseler, Jörg

Herold, Sven

Heyne, Joshua

Highhouse, Scott

Hofer, Matthias

Hollensbe, Elaine

Hondori, Hossein

Hošovský, Alexander

$\mathrm{Hu}, \mathrm{Kejia}$

$\mathrm{Hu}$, Shu-Kung

Hunstig, Matthias

Hur, Deog Jae

Irving, Justin

Jang, Sung-Hwan

Janousek, Christian
Jaskyte, Kristina

Jastrzebski, Rafal

Jha, Chandan K.

Jiang, Dong

Jones, Karen

Jugo, Josu

Junjan, Veronica

Kaal, William

Kadumudi, Firoz Babu

Kanda, Takefumi

Kang, Miliann

Kermani, Mehrdad R.

Kiefer, Rudolf

Kim, EunHee

Kim, Yeongjin

Kimmerle, Joachim

Kimura, Shinichi

Kisilowski, Maciej

Kluemper, Don

Kniesburges, Stefan

Knospe, Carl

Kolomenskiy, Dmitry

Komori, Mochimitsu

Kong, Ling Bing

Kotilainen, Sirkku

Kouh, Taejoon

Kricheli-Katz, Tamar

Krueger, Joachim

Kumaran, Muthusami

Kurucz, Elizabeth C.

Lacroix, Martin

LaHaye, Matthew

Lai, Gavin

Lam, Garret C.Y.

Landry, Anaïs Thibault

Lasson, Elliot

lastname, firstname

Lau, Fernando

Laub, Jim

Laumer, Sven

Leavitt, Lynda

Lee, Allan

Lee, Jae Shin 
Lee, Mikyoung

Lee, Yong Bok

Lev, Dan

Levasseur, Karine

Li, Shiyang

Liou, James J. H.

Lisa, Carbone

Liu, Suqin

Liu, Tzong-shi

Lofgren, Karl

Loftin, Collette

Maas, Jürgen

MacPhee, Maura

Madsen, Susan R

Marcy, Richard

Mariani, Raffaello

Marle, Franck

Martin, Jennifer

Mavris, Dimitri

Mayers, Raymond Sanchez

Mcpake, Barbara

Mease, Jennifer

Merola, Alessio

Minerbi, Luciano

Misgeld, Berno

Molina-Morales, Francesc Xavier

Molloy, Timothy

Moorosi, Pontso

Moriello, Lorenzo

Mothersell, William

Mueller, Torsten

Müller, Bert

Murrenhoff, Hubertus

Mystkowski, Arkadiusz

Nair, Girija

Narooz, Rose

Naso, David

Nguyen, Tam

Nicholas, John

Noda, Ryusuke

Noordegraaf, Mirko

Nurmi, Jarmo

Olafsen, Anja Hagen
Oldham, Kenn

Ongaro, Edoardo

Pantelis, Nikolakopoulos

Park, Sangchan

Patterson, Kathleen

Pereira, Dolores

Perera, Luckmika

Pesch, Alexander H.

Pircher Verdorfer, Armin

Pitel', Ján

Plooij, Michiel

Plummer, Virginia

Pott, Peter

Pręgowska, Agnieszka

Psychogios, Alexandros

Purdy, Nancy

Rabenorosoa, Kanty

Ragusett, Jared

Rakotondrabe, Micky

Ramasamy, Subramanian

Ran, Bing

Raper, David

Rauch, Erwin

Reddy, Sohail

Rekveldt, Marian Bosch

Remke, Dr. Robyn

Ristic, Michael

Rohacs, Jozsef

Rosendo, Andre

Rosenow, Judith

Roy, Michael J

Ryan, Susan

Saadeh, Mohammad

Sadler-Smith, Eugene

Salas, Jesus M.

Sanga, Kabini

Scannapieco, Antonio Fulvio

Schneider, Ben $\mathrm{H}$.

Selck, Torsten J.

Semlitsch, Bernhard

Sermeus, Walter

Serpa, Sandro

Serpilli, Michele 
Shaw, Alexander

Shiao, Yaojung

Siemienska, Renata

Silvestre, Miguel

Singh, Mina

Siqueira, Adriano

Sivasubramanian, Jayahar

Skidmore, Jeffrey

Sloan, Margaret

Sohmen, Victor

Sousa, Carlos M.P.

Spicer, David

Stanley, Andrew A.

Stephenson, Max O.

Strydom, Reuben

Stump, Erika

Su, Chun-yi

$\mathrm{Su}$, Weihua

Sujit, P.B

Sun, Guanghui

Suzumori, Koichi

Tadrist, Loïc

Takosoglu, Jakub Emanuel

Talat, Usman

Tarisciotti, Luca

Tomazevic, Nina

Tseng, Ming-Lang

Tundys, Blanka

Turkoglu, Kamran

Tzanakou, Charoula

Uchiyama, Kenji

Udod, Sonia
Uekermann, Benjamin

Urzi Brancati, Cesira

Van Der Steen, Martijn

Van Dierendonck, Dirk

Vanderborght, Bram

Venkatraman, Sitalakshmi

Vespe, Francesco

Wagner, Joan

Wang, Shiqian

Warren, Clive

Warren, Lorraine

Weaver, Andrew

Weber, Robert

Wells, Paul

Wharton, Amy

White, Kate

Wierenga, Berend

Wollbrant, Conny

Wong, Vincent

Woodhouse, Joan

$\mathrm{Wu}$, Jiagang

Yakinthos, Kyros

Yao, YuChun

Yeow, Chen Hua

Yousefi, Kianoosh

Zhan, Haifei

Zhang, Dou

Zhao, Huichan

Zhao, Qiuying

Zhao, Xiaohui

Zhou, Jin

Zhu, George Z.H.

(C) 2018 by the author. Submitted for possible open access publication under the terms and conditions of the Creative Commons Attribution (CC-BY) license (http://creativecommons.org/licenses/by/4.0/). 